\title{
Cortical actin flow activates an $\alpha$-catenin clutch to assemble adherens
}

\section{junctions}

\author{
Authors: Ivar Noordstra ${ }^{1}$, Mario Díez Hermoso ${ }^{2}$, Lilian Schimmel ${ }^{1}$, Alexis Bonfim-Melo ${ }^{1}$, \\ Joseph Mathew Kalappurakkal ${ }^{3,5}$, Satyajit Mayor ${ }^{3}$, Emma Gordon ${ }^{1}$, Pere Roca Cusachs ${ }^{2,4,6}$ and \\ Alpha S. Yap ${ }^{1,6}$
}

\section{Affiliations:}

${ }^{1}$ Division of Cell and Developmental Biology, Institute for Molecular Bioscience, The University of Queensland; St. Lucia, Brisbane, Queensland, Australia 4072

${ }^{2}$ Institute for Bioengineering of Catalonia (IBEC), the Barcelona Institute of Technology (BIST); 08028 Barcelona, Spain

${ }^{3}$ National Centre for Biological Science, Tata Institute for Fundamental Research; Bangalore, 560065, India

${ }^{4}$ Universitat de Barcelona; 08036 Barcelona, Spain

${ }^{5}$ Present address: Department of Physiology, Development and Neuroscience, University of Cambridge; Cambridge, CB2 3EG, United Kingdom

${ }^{6}$ Correspondence: a.yap@uq.edu.au (experimental) and proca@ibecbarcelona.eu (theory)

\begin{abstract}
Adherens junctions (AJs) fundamentally mediate cell-cell adhesion, yet the mechanisms that determine where or when AJs assemble are not understood. Here we reveal a mechanosensitive clutch that initiates AJ assembly. Before cell-cell contact, $\alpha$-catenin couples surface E-cadherin complexes to retrograde flow of the actin cortex. Cortical flows with opposed
\end{abstract}


orientations persist after contact, applying tension to $\alpha$-catenin within trans-ligated cadherin complexes. Tension unfolds the $\alpha$-catenin actin-binding domain (ABD), which is expected to mediate a catch bond with F-actin. However, catch bond behaviour is not sufficient for AJ assembly in a molecular clutch model. Instead, it is also necessary for the activated ABD to promote cis-clustering of E-cadherin molecules by bundling F-actin. Thus, this $\alpha$-catenin clutch transduces the mechanical signal of cortical flow to assemble AJs.

Main Text: The assembly of adherens junctions (AJ) is a fundamental determinant of tissue organization and homeostasis during development and in post-embryonic life $(1,2)$. AJ promote cell-cell cohesion and regulate motility, proliferation and cell differentiation. AJ constitute multimolecular membrane platforms that are built upon classical cadherin adhesion receptors. But while AJs require the adhesive trans-ligation of their cadherins, how cadherin engagement leads to AJ assembly remains poorly understood. There is ample evidence that AJ do not assemble as an obligate consequence of cadherin trans-ligation. For example, cadherins are already present on cell surfaces before AJ form during mouse embryo compaction (3) and Drosophila cellularization (4). Nor can trans-ligated cadherins support robust cell-cell adhesion if they cannot engage catenins (5-7). Consequently, it has often been inferred that the cadherin apparatus must be activated for AJs to assemble (8-12), but how this occurs is not known. A distinct possibility is that cells generate mechanical signals when they make contact with one another that provide the stimulus for AJ assembly $(13,14)$. But the source of the mechanical force for such a signal, and the mechanism that it engages to assemble AJ, remain unknown. 
AJs assemble from surface cadherins coupled to cortical actin flow. To investigate how cells commit to assembling AJs, we monitored migrating MCF7 mammary epithelial cells bearing endogenous E-cadherin tagged with GFP by CRISPR/Cas9 gene-editing (E-cadherin-GFP ${ }^{\text {CRISPR }}$ ). Cells displayed prominent lamellipodia (detected with co-expressed LifeAct) as they migrated in artificial wound assays and contacted each another when their lamellipodia touched (Fig 1A). Some cells retracted and broke contact almost immediately after this initial contact, whereas in other cells overlapping contacts persisted to become the sites where intense E-cadherin-

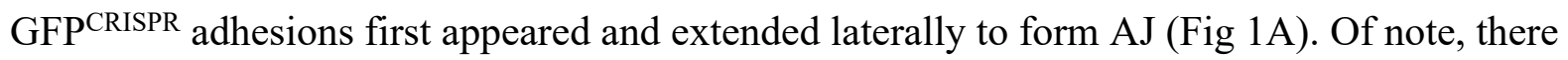
was a consistent 10 - 15 min delay from when cells first contacted one another to AJs appearing (Fig 1B; movie S1). During this period E-cadherin-GFPCRISPR levels increased with characteristically sigmoidal kinetics, to plateau with the first AJs (Fig 1B, C). This suggested that a specific mechanism operates in this period that commits cells to assemble AJ.

To understand how these AJ assemble, we imaged E-cadherin-GFP in living cells before they made contact with one another. E-cadherin was found in prominent punctate structures that appeared identical with E-cadherin-GFPCRISPR (Fig S1A) or with an exogenously-expressed transgene (E-cadherin-GFP ${ }^{\mathrm{TG}}$ ) (Fig 1D). The greater signal strength of E-cadherin-GFP ${ }^{\mathrm{TG}}$ revealed that these puncta formed two populations with distinct patterns of movement. One population showed rapid $(\sim 1.5 \mu \mathrm{m} / \mathrm{min})$, saltatory movements in both anterograde and retrograde directions (Fig 1D, E, yellow; movie S2); often appeared to move along microtubules (Fig S1B; movie S3); and were not labelled with extracellular E-cadherin mAbs (Fig 1D, yellow; movie S2). We consider these to be E-cadherin-containing intracellular vesicles.

In contrast, $\sim 50 \%$ of E-cadherin-GFP ${ }^{\mathrm{TG}}$ was found in puncta that underwent a distinctive, slow ( $\sim 0.2 \mu \mathrm{m} / \mathrm{min})$, processive retrograde movement (Fig 1D, E, red; movie S2). These could 
be co-labelled in live cells with extracellular E-cadherin mAbs (Fig 1D, red; movie S2), but their size was not altered by the mAbs, indicating that they were not due to mAb-induced crosslinking. These slow-moving puncta therefore appeared to represent E-cadherin cis-clusters on the free surfaces of cells, as have been previously reported $(15,16)$. Characteristically, surface cisclusters formed when more diffuse E-cadherin coalesced at the leading edges of the lamellipodia (Fig S1C; movie S4). As lamellipodia are classic sites of retrograde actin flow (17), this suggested that cortical flow might drive the movement of surface E-cadherin clusters. Indeed, a drug cocktail (of blebbistatin, CK666, jasplakinolide) that effectively suppressed retrograde actin flow also abolished retrograde transport of surface E-cadherin (Fig 1F, G, S1D; movie S5). one another $($ Fig $1 \mathrm{H})$, but we did not detect entry of rapidly-moving puncta that would represent delivery of cadherin from intracellular vesicles. This indicated that AJs are assembled from surface cadherins which are already associated with the flowing actin cortex. indicated a physical association between the cadherins and cortical F-actin. This may have been mediated by catenins that are present in the surface E-cadherin clusters (Fig 2A) or due to nonspecific interactions with the cortex. To distinguish these possibilities, we targetted $\alpha$-catenin, which links the core cadherin-catenin complex to F-actin $(18,19)$. We first endeavoured to test the effects of deleting $\alpha$-catenin by CRISPR/Cas9 ( $\alpha$-catenin KO) (Fig S2A-D). Although substantial amounts of surface E-cadherin were retained in $\alpha$-catenin $\mathrm{KO}$ cells (Fig S2E), cisclusters were reduced, making it difficult to measure cadherin flow (Fig S2F-H; movie S6). 
Instead, we reconstituted $\alpha$-catenin $\mathrm{KO}$ cells with an $\alpha$-catenin transgene $\left(\alpha\right.$-catenin $\left.{ }^{\mathrm{A}+}\right)$

(Fig S3A) that has increased affinity for actin filaments, because an intramolecular interaction in the actin-binding domain (ABD) is disrupted (19). Surface E-cadherin transport was $\sim 8 x$ slower than retrograde cortical flow in cells that express WT $\alpha$-catenin, either controls or KO cells reconstituted with an $\alpha$-catenin ${ }^{\mathrm{WT}}$ transgene (Fig 1E, 2C). In contrast, reconstitution with the higher-affinity $\alpha$-catenin $^{\mathrm{A}+}$ transgene, which incorporated into free cadherin clusters (Fig 2B), significantly increased the speed of cadherin transport without affecting cortical actin flow itself (Fig 2B, C). This strongly suggests that there is slippage in the linkage between $\alpha$-catenin and Factin which is reduced by $\alpha$-catenin ${ }^{\mathrm{A}+}$. Therefore, $\alpha$-catenin likely directly couples the core E10 cadherin-catenin complex on free cell surfaces to the actin cortex.

Trans-ligated cadherins engage with cortical flow. Direct coupling to the cortex could poise unligated cadherin complexes to assemble AJ upon cell-cell contact. Therefore, we asked how AJ assembly was affected when cadherins were uncoupled from the cortex by $\alpha$-catenin KO. In contrast to WT controls, $\alpha$-catenin KO cells always retracted after they touched, manifest as a saw-tooth pattern of contact in kymographs, and failed to form AJ even at confluence (Fig 2D, $\mathrm{S} 2 \mathrm{C}$; movie S7). While this suggested a role for cortical coupling, the inability of $\alpha$-catenin $\mathrm{KO}$ cells to generate cadherin cis-clusters could also have explained the defect in AJ assembly. We resolved these possibilities by studying heterologous interactions between $\mathrm{WT}$ and $\alpha$-catenin $\mathrm{KO}$ cells (Fig 2D, E). Here cadherins in the $\alpha$-catenin KO cells formed clusters at the overlaps with the WT cells (Fig 2E; movie S8). Presumably, these reflect trans-ligation of cadherins in the KO cells with clustered cadherins in the WT cells. But they never accumulated to form AJs, nor did AJs form between heterologous pairs of WT and $\alpha$-catenin KO cells, even in confluent cultures 
(Fig 2D; movie S7). Therefore, the ability to cluster trans-ligated cadherins was not sufficient for AJ assembly. By implication, another process must operate after trans-ligation that activates the cadherin system to assemble AJs.

Insight into what this may be came from visualizing the dynamics of cadherin and the actin cortex when cells first made contacts with one another. Strikingly, cortical flow was evident in lamellipodia even after they made contact with one another (Fig 2F; movie S9). Indeed, it only stopped when E-cadherin levels plateaued to form AJs (Fig 2G). Furthermore, the cortical flows in overlapping cells were organized in an antiparallel orientation (Fig 2F; movie S9) that would be expected to cause the flows to oppose one another when coupled together by cadherin trans-ligation. In contrast, flows did not stop when $\alpha$-catenin $\mathrm{KO}$ cells made contact with WT cells, even though these heterologous contacts were capable of trans-ligating Ecadherin (Fig 2D, E, S3B; movie S7). Moreover, cadherin clusters in the $\alpha$-catenin KO cells were transported in an anterograde direction at these heterologous contacts (Fig 2E; movie S8). This suggested that trans-ligated cadherin clusters uncoupled from the cortex in the $\alpha$-catenin KO cells were being dragged by retrograde transport of cadherin in the WT cell. Together, these data indicated that coupling to the cortex subjects trans-ligated cadherin clusters to anti-parallel flows when cells make contact and assemble AJs.

Adhesive load allows cortical flow to activate the cadherin-catenin complex. Cortical flows can generate mechanical force $(20,21)$, which would be enhanced by the opposed, antiparallel orientation that accompanied AJ assembly. This led us to hypothesize that cortical flow generates a mechanical signal to initiate junction assembly. Specifically, we considered that cortical flow would only exert minor forces on cadherins that are flowing on the free surface, where resistance 
to flow is minimal. But these forces would be increased upon contact when cadherin ligation coupled the opposed flows of neighbour cells together to exert load upon each other, leading to activation of some element(s) of the cadherin molecular complex.

This hypothesis required that cortical flow generate forces strong enough to alter the cadherin-catenin complex upon adhesive load. We tested this by examining the interaction between cells and substrata coated with E-cad ectodomain ligands (Ecad-Fc) (Fig S4A). This allowed us to analyze zones of adhesive trans-ligation with high temporal and spatial resolution. Under these conditions, cellular E-cadherin is immobilized and retrograde cortical F-actin flow persists at the cell-substrate interface, being characteristically found in the lamella of cells spreading on E-cadherin, but not in the area beneath nuclei (Fig 3A; movie S10). We then used soft PDMS substrata containing beads to detect the pattern of traction forces applied across Ecadherin by the cells. This revealed a periodic displacement of the beads, indicating the application of mechanical force, that coincided exactly with waves of F-actin that passed over the beads (Fig 3B, C). catenin complex, we interrogated $\alpha$-catenin, which undergoes conformational unfolding when tension is applied to the cadherin molecular complex $(19,22)$. One of these changes is reported by the $\alpha 18 \mathrm{mAB}$, which recognizes a force-sensitive epitope in the central M-domain (22). $\alpha 18$ mAB stained precisely in the lamella where actin flow was found, but not in the subnuclear area of the cells, where flow was absent (Fig 3D, F). This was confirmed using a FRET-based $\alpha$ catenin conformation sensor (Fig 3E, F) (23). Importantly, cortical flow was the source of mechanical force, as these conformational responses were reversed when flow was blocked (Fig 3D, E, G). Nor was conformational change observed at cis-clusters in the lamellae of cells plated 
Mechanical activation of $\alpha$-catenin promotes AJ assembly. Could this mechanical impact on $\alpha$ catenin have been responsible for initiating AJ assembly? Mechanical tension is known to open the central M domain $\alpha$-catenin to promote association with vinculin (22), and also unfolds the autoinhibited alphal helix in the F-actin-binding site (ABD) to increase its affinity for actin filaments (19). To evaluate which of these might influence AJ assembly, we reconstituted $\alpha$ catenin KO cells with either a vinculin-uncoupled mutant (V-) (Fig S3A) (24), used to test whether there was a role for the molecular consequence of M-domain unfolding; or with mutants in the ABD which either ablate F-actin binding (A-) or that unfold the ABD to increase its affinity $(\mathrm{A}+)(19)\left(\right.$ Fig S3A). Cells reconstituted with $\alpha$-catenin ${ }^{\mathrm{A}-}$ failed to assemble AJ when grown to confluence, but junctions were restored with $\alpha$-catenin ${ }^{\mathrm{A}+}(\mathrm{Fig} 4 \mathrm{~A})$. This implied that Factin binding was necessary for AJ assembly. In contrast, AJ were effectively restored with $\alpha$ catenin $^{\mathrm{V}-}$ (Fig 4A), implying that the M-domain mechanosensitivity responsible for recruiting vinculin was dispensable for AJ assembly.

This was confirmed by evaluating the initiation of AJ assembly. Expression of $\alpha$ catenin $^{\mathrm{V}-}$ at levels which displace vinculin from AJ (Fig S4D, E) did not alter the kinetics of AJ assembly (Fig 4B, C). In contrast, AJ assembly was dramatically accelerated by the expression of $\alpha$-catenin ${ }^{\mathrm{A}+}$ (Fig 4B, C). (The $\alpha$-catenin ${ }^{\mathrm{A}-}$ mutant was not tested, because the cells did not form AJ.) As the $\alpha$-catenin ${ }^{\mathrm{A}+}$ mutant reproduces the molecular effect of applying tension to the 
$\mathrm{ABD}$, this indicated that mechanical activation of the ABD was sufficient to initiate $\mathrm{AJ}$ assembly.

The $\alpha$-catenin clutch is more than a catch bond. To understand what would be required for ABD activation to initiate $\mathrm{AJ}$ assembly, we then modelled the cadherin-catenin complex as a mechanosensitive clutch where tension alters the binding properties of the ABD (detailed in the Computational Supplement). Here, we define a "clutch" as a pre-coupled complex of E-cadherin and $\alpha$-catenin, trans-ligated to an equivalent complex on the other cell once the cells make contact, and to the actin cytoskeleton also on both sides (Fig S5A). Upon cell-cell contact, initial adhesions are thus composed of a given number of bound clutches, which get submitted to force as actin pulls in opposite directions from both cells due to the antiparallel actin flows. Force exerted on clutches has two effects: first, it can lead to unfolding of the alpha1 helix, with an unfolding rate that depends on force as a slip bond (19). Second, it affects the unbinding rate between $\alpha$-catenin and actin, which depends on force as either a slip bond (if the alpha1 helix is folded) or a catch bond (if it is unfolded) (19). Once clutches unbind, they can rebind again according to a given binding rate.

As expected, force transmission to the clutches led to alpha1 helix unfolding, strengthening bonds to actin through catch bonds, and thereby increasing resistance to actin flow. This led to a small decrease in actin flows (Fig S5B, C). But this occurred over time scales of seconds and did not yield the minutes-scale adhesion growth that we observed experimentally. This was because, based on the reported $\mathrm{K}_{\text {off }}$ for $\alpha$-catenin and F-actin (18), even in their highaffinity state clutches were predicted to disengage from F-actin on seconds-scales and thus fail to 
sustain cadherin growth over longer scales. This implied that the $\alpha$-catenin-actin catch bond was not sufficient to explain how the cadherin clutch promotes AJ assembly.

We therefore considered other potential mechanisms that could be engaged when the alpha1 helix is unfolded. Of note, unfolding of the alpha1 helix supports F-actin bundling as well as increasing binding affinity (19). By bringing together F-actin filaments that anchor cadherin clutches, this could increase the number of cadherin-catenin complexes that are incorporated into clusters. Further, force-induced actin bundling is also observed in the context of cell-matrix adhesion (25), and its timescale (of the order of minutes rather than seconds) matches well what we observed for AJ. To model this, we incorporated growth of cadherin clusters as a consequence of tension-induced alpha1 helix unfolding. Under these conditions, tensiondependent engagement of cadherin clutches yielded growth of cadherin junctions, with a sigmoidal profile and minutes-scale time course that closely resembled what we observed experimentally (Fig 4C, D, S5D). Consistently, actin flows also progressively slowed down within the same time scale (Fig. 4E). Moreover, the effects on adhesion growth and actin flows were dramatically accelerated when we modelled the $\alpha$-catenin ${ }^{A+}$ mutant as a clutch with the alpha1 helix locked in its open conformation (Fig 4D, 4F, S5E). This also paralleled exactly what we had observed experimentally (Fig 4C). Therefore, AJ assembly in the model required that the $\alpha$-catenin clutch promote cadherin cis-clustering, as well as increase affinity for F-actin.

To test this prediction, we analysed how the $\alpha$-catenin ${ }^{\mathrm{A}+} \mathrm{ABD}$ mutation affected cadherin cis-clusters at the free surfaces of cells. This experimental design was used to isolate the impact of altering $\alpha$-catenin, without other confounding effects associated with cadherin ligation. We predicted that $\alpha$-catenin ${ }^{\mathrm{A}+}$ should increase the incorporation of E-cadherin into clusters, even without the mechanical loading that comes from homophilic trans-ligation. Expressing $\alpha$ - 
catenin $^{\mathrm{A}+}$ in $\alpha$-catenin $\mathrm{KO}$ cells did not alter the size of surface cadherin clusters detectable with diffraction-limited optics. However, $\alpha$-catenin ${ }^{\mathrm{A}+}$ significantly increased E-cadherin fluorescence intensity of the clusters (Fig 4G, H), consistent with enhanced recruitment of E-cadherin molecules. Then, we further mutated $\alpha$-catenin ${ }^{\mathrm{A}+}$ to delete a region in the $\operatorname{ABD}(\alpha$-catenin ${ }^{\mathrm{A}+\Delta \beta \mathrm{H}}$ ) that is implicated in bundling actin filaments (19) (Fig S3A). This abrogated the ability of $\alpha$-catenin ${ }^{\mathrm{A}+}$ to promote cadherin clustering (Fig 4G, H). Furthermore, although $\alpha$-catenin ${ }^{\mathrm{A}+/ \Delta \beta \mathrm{H}}$ cells formed contacts with one another, they did not display the linear AJ characteristic of WT cells (Fig 4A). Therefore, in addition to conferring catch-bond properties on its association with F-actin, unfolding of the alphal helix in its ABD allows $\alpha$-catenin to promote cis-clustering of E-cadherin through actin bundling. This also explains why cis-clusters failed to form in $\alpha$ catenin $\mathrm{KO}$ cells.

Our findings thus reveal how a tension-sensitive molecular clutch activates cadherin adhesion to assemble AJs. There is a beauty in the simplicity of this mechanism. Prior association of the cadherin-catenin complex with the actin cortex means that tension can be immediately exerted upon $\alpha$-catenin when adhesive trans-ligation couples the flows of contacting cells together. Force-induced unfolding of the $\alpha 1$ helix in the ABD then initiates AJ assembly by enhancing cis-clustering of cadherins, a previously unknown function of this domain that is mediated by enhanced F-actin bundling (Fig 4I). While many other cytoskeletal and signaling events contribute to $\mathrm{AJ}$ maturation $(9,26,27)$, we propose that this $\alpha$-catenin clutch is the gatekeeper that first triggers the assembly process. This mechanism can inform how cells choose contacts to assemble $\mathrm{AJ}$, as we found that the coupling of opposed cortical flows was necessary to activate the $\alpha$-catenin clutch. Oppositely-directed cortical flows persisted until AJs formed and neither drag forces from the membrane or adhesion forces from trans-ligation 
could induce AJ assembly if cadherins were uncoupled from the cortex on even one side of a contact. Cells may then assemble AJs at contacts where cadherin trans-ligation allows opposed cytoskeletal forces to generate a mechanical signal that is strong enough to activate the $\alpha$-catenin clutch. We identified this for the cortical flows of lamellipodia in migrating cells and it may generalize when other sources of opposed force are brought together by cadherin trans-ligation $(28,29)$.

\section{References}

1. T. J. Harris, U. Tepass, Adherens junctions: from molecules to morphogenesis. Nat Rev Mol Cell Biol 11, 502-514 (2010).

2. C. Niessen, D. Leckband, A. S. Yap, Tissue organization by classical cadherin adhesion molecules: dynamic molecular and cellular mechanisms of morphogenetic regulation. Physiol Rev 91, 691-731 (2011).

3. D. Vestweber, A. Gossler, K. Boller, R. Kemler, Expression and distribution of cell adhesion molecule uvomorulin in mouse preimplantation embryos. Developmental Biology 124, 451456 (1987).

4. T. J. Harris, M. Peifer, Adherens junction-dependent and -independent steps in the establishment of epithelial cell polarity in Drosophila. J Cell Biol 167, 135-147 (2004).

5. M. Watabe, A. Nagafuchi, S. Tsukita, M. Takeichi, Induction of polarized cell-cell association and retardation of growth by activation of the E-cadherin-catenin adhesion system in a dispersed carcinoma line. J. Cell Biology 127, 247-256 (1994). 
6. M. Ozawa, M. Ringwald, R. Kemler, Uvomorulin-catenin complex formation is regulated by a specific domain in the cytoplasmic region of the cell adhesion molecule. Proceedings of the National Academy of Sciences, USA 87, 4246-4250 (1990).

7. A. Nagafuchi, M. Takeichi, Cell binding function of E-cadherin is regulated by the cytoplasmic domain. EMBO J 7, 3679-3684 (1988).

8. T. P. Fleming, M. R. Ghassemifar, B. Sheth, Junctional complexes in the early mammalian embryo. Semin Reprod Med 18, 185-193 (2000).

9. R. M. Mege, N. Ishiyama, Integration of Cadherin Adhesion and Cytoskeleton at Adherens Junctions. Cold Spring Harb Perspect Biol 9, (2017).

10. B. A. Truong Quang, M. Mani, O. Markova, T. Lecuit, P. F. Lenne, Principles of E-cadherin supramolecular organization in vivo. Current biology : CB 23, 2197-2207 (2013).

11. B. M. Gumbiner, Regulation of cadherin adhesive activity. J. Cell Biol. 148, 399-403 (2000).

12. J. L. Maitre, C. P. Heisenberg, Three functions of cadherins in cell adhesion. Current biology : $C B$ 23, R626-633 (2013). Dev Biol 30, 291-315 (2014).

14. B. D. Hoffman, A. S. Yap, Towards a Dynamic Understanding of Cadherin-Based Mechanobiology. Trends Cell Biol 25, 803-814 (2015).

15. A. Padmanabhan, H. T. Ong, R. Zaidel-Bar, Non-junctional E-Cadherin Clusters Regulate the Actomyosin Cortex in the C. elegans Zygote. Current biology : CB 27, 103-112 (2017). 
16. R. Chandran, G. Kale, J. M. Philippe, T. Lecuit, S. Mayor, Distinct actin-dependent nanoscale assemblies underlie the dynamic and hierarchical organization of E-cadherin. Current biology : CB 31, 1726-1736 e1724 (2021).

17. P. Vallotton, S. L. Gupton, C. M. Waterman-Storer, G. Danuser, Simultaneous mapping of filamentous actin flow and turnover in migrating cells by quantitative fluorescent speckle microscopy. Proceedings of the National Academy of Sciences of the United States of America, (2004).

18. C. D. Buckley et al., The minimal cadherin-catenin complex binds to actin filaments under force. Science 346, 1254211-1254211-1254218 (2014).

19. N. Ishiyama et al., Force-dependent allostery of the alpha-catenin actin-binding domain controls adherens junction dynamics and functions. Nat Commun 9, 5121 (2018).

20. N. W. Goehring et al., Polarization of PAR proteins by advective triggering of a patternforming system. Science 334, 1137-1141 (2011).

21. A. Reversat et al., Cellular locomotion using environmental topography. Nature 582, 582585 (2020).

22. S. Yonemura, Y. Wada, T. Watanabe, A. Nagafuchi, M. Shibata, alpha-Catenin as a tension transducer that induces adherens junction development. Nat Cell Biol 12, 533-542 (2010).

23. T. J. Kim et al., Dynamic Visualization of alpha-Catenin Reveals Rapid, Reversible Conformation Switching between Tension States. Current biology : CB 25, 218-224 (2015). mechanism to activate vinculin. J Biol Chem 287, 7728-7737 (2012). 
25. P. W. Oakes et al., Optogenetic control of RhoA reveals zyxin-mediated elasticity of stress fibres. Nat Commun 8, 15817 (2017).

26. W. Meng, M. Takeichi, Adherens junction: molecular architecture and regulation. Cold Spring Harb Perspect Biol 1, a002899 (2009).

27. A. S. Yap, G. A. Gomez, R. G. Parton, Adherens Junctions Revisualized: Organizing Cadherins as Nanoassemblies. Developmental cell 35, 12-20 (2015).

28. S. Zhang, X. Teng, Y. Toyama, T. E. Saunders, Periodic Oscillations of Myosin-II Mechanically Proofread Cell-Cell Connections to Ensure Robust Formation of the Cardiac Vessel. Current biology : CB 30, 3364-3377 e3364 (2020).

29. J. L. Maitre et al., Adhesion functions in cell sorting by mechanically coupling the cortices of adhering cells. Science 338, 253-256 (2012).

30. X. Liang et al., Tyrosine dephosphorylated cortactin downregulates contractility at the epithelial zonula adherens through SRGAP1. Nat Commun 8, 790 (2017).

31. A. Elosegui-Artola et al., Mechanical regulation of a molecular clutch defines force transmission and transduction in response to matrix rigidity. Nat Cell Biol 18, 540-548 (2016).

32. Y. Wu, P. Kanchanawong, R. Zaidel-Bar, Actin-delimited adhesion-independent clustering of E-cadherin forms the nanoscale building blocks of adherens junctions. Dev Cell 32, 139$154(2015)$ types. Nat Mater 13, 631-637 (2014). 
34. J. E. Molloy, J. E. Burns, J. Kendrick-Jones, R. T. Tregear, D. C. White, Movement and force produced by a single myosin head. Nature 378, 209-212 (1995).

35. S. Rakshit, Y. Zhang, K. Manibog, O. Shafraz, S. Sivasankar, Ideal, catch, and slip bonds in cadherin adhesion. Proc Natl Acad Sci U S A 109, 18815-18820 (2012).

Acknowledgments: We thank Srikanth Budnar for the E-cadherin-GFPCRISPR cell line, Denni Currin-Ross for curve fitting, and all our colleagues in the lab for their support, advice and encouragement. Microscopy was performed at the ACRF/IMB Cancer Research Imaging Facility created with the generous support of the Australian Cancer Research Foundation.

\section{Funding:}

National Health and Medical Research Council of Australia (project grants GNT1140090, GNT1154462 and fellowship GNT1136592 to A.S.Y.; Project grant APP1158002 to E.G.).

Australian Research Council (DP19010287 to A.Y.; DP200100737 to E.G.).

National Heart Foundation of Australia: E.G. was supported by a Future Leader

Fellowship - Level 2 (104692) from the National Heart Foundation of Australia.

European Molecular Biology Organization: I.N. was supported by EMBO ALTF 2512018. (UQECR2058733). 
Spanish Ministry of Science and Innovation: P.R.-C. (PID2019-110298GB-I00, PGC2018-099645-B-I00).

European Commission: P.R.-C. (H2020-FETPROACT-01-2016-731957)

Generalitat de Catalunya: P.R.-C. (2017-SGR-1602).

Fundació la Marató de TV3: P.R.-C. (201936-30-31).

The prize "ICREA Academia" for excellence in and "la Caixa" Foundation: P.R.-C. (Agreement LCF/PR/HR20/52400004).

Government of India: S.M. was supported by JC Bose Fellowship from DST and Margadarshi fellowship (IA/M/15/1/502018).

\section{Author contributions:}

Conceptualization: I.N., P.R.-C., A.S.Y.

Methodology: I.N., M.D.H., P.R.-C.

Formal analysis: I.N., M.D.H.

Investigation: I.N., L.S., A.B-M.

Writing: I.N., M.D.H., J.M.K., S.M., E.G., P.R.-C., A.S.Y.

Visualization: I.N., M.D.H.

Supervision: P.R.-C., A.S.Y.

Funding acquisition: I.N., L.S., E.G., P.R.-C., A.S.Y. 
bioRxiv preprint doi: https://doi.org/10.1101/2021.07.28.454239; this version posted July 28, 2021. The copyright holder for this preprint (which

was not certified by peer review) is the author/funder, who has granted bioRxiv a license to display the preprint in perpetuity. It is made available under aCC-BY-NC-ND 4.0 International license.

Data and materials availability: All data are available in the main text or the supplementary materials.

\section{Supplemental Material}

Materials and Methods

5 Computational Supplement

Figs. S1 to S6

Tables S1 and S2

Movies S1 to S10 

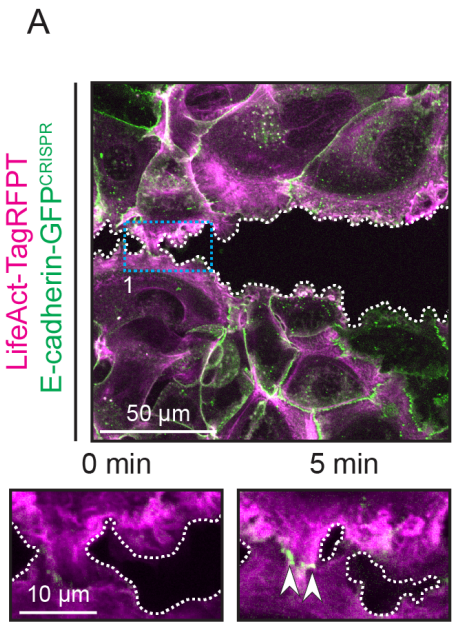

15 min
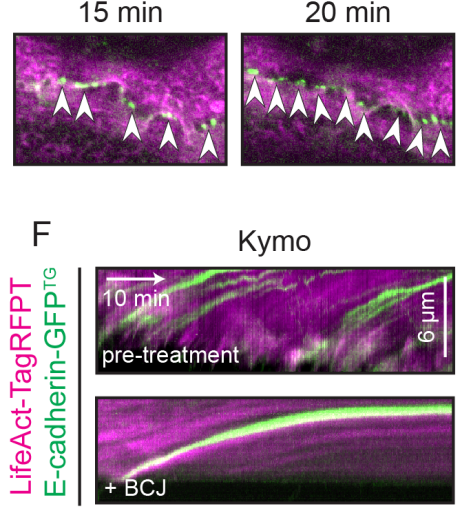

G

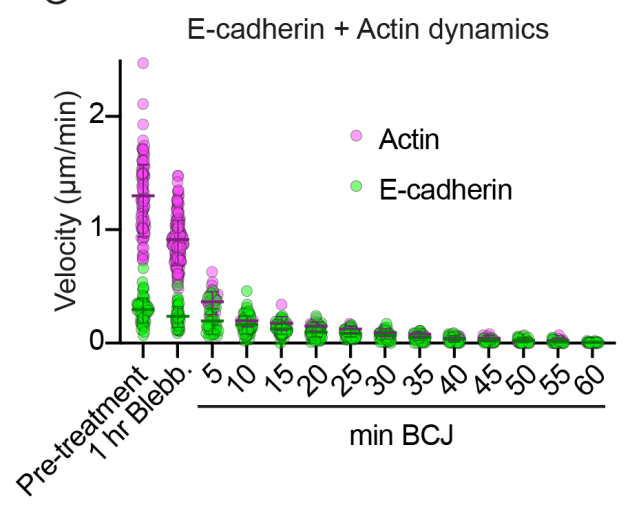

B

D
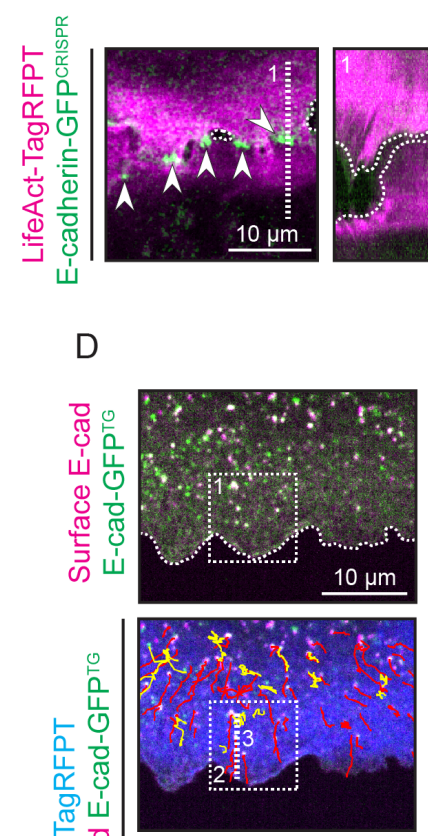

Kymo

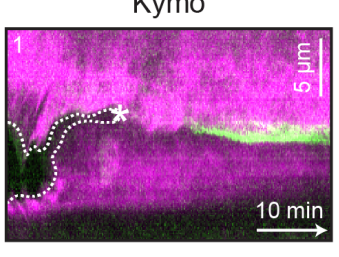

C

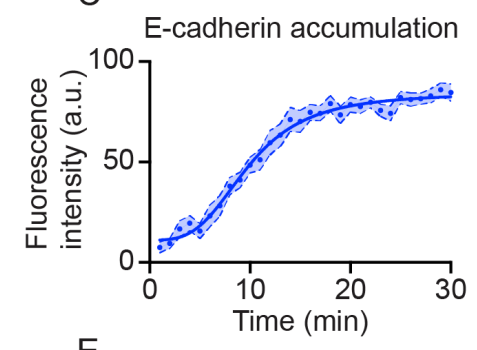

E
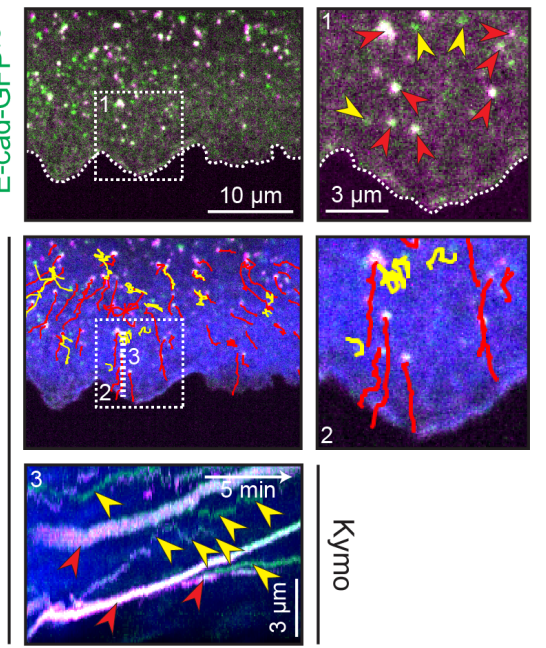

$\mathrm{H}$
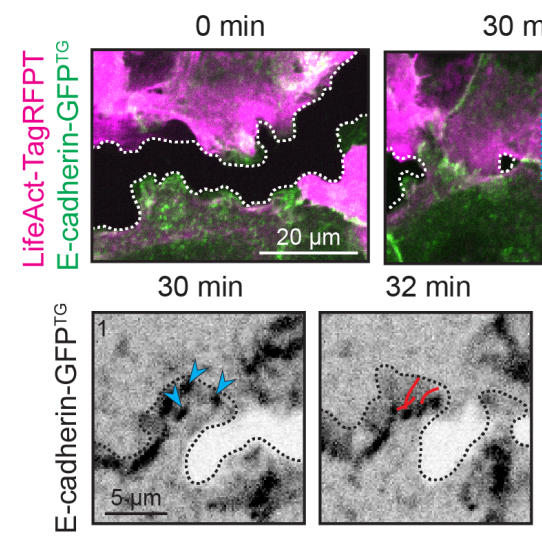

E-cadherin + Actin dynamics

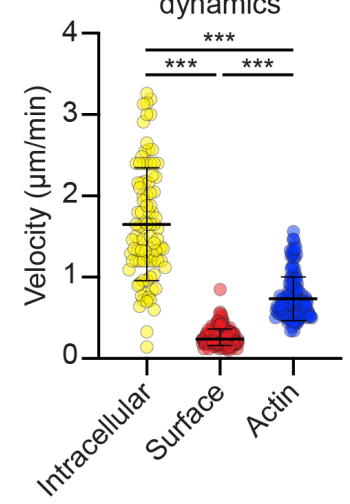

\section{Figure 1. Adherens junctions are initiated from surface E-cadherin that is coupled to}

\section{cortical flow.}


(A) Junction formation between MCF7 cells expressing LifeAct-TagRFPT (magenta) and Ecadherin-GFPCRISPR (green). White dotted line: free cell edge; arrow heads: Adherens Junctions $(\mathrm{AJ})$.

(B) Spatiotemporal dynamics of AJ formation in cells as in (A). Arrow heads: AJ; wavy white dotted line: free cell edge; straight white dotted line: site of kymograph; *First moment of contact.

(C) Quantification of E-cadherin intensity in cells as in (B). Junction formation events ( $\mathrm{n}=13)$. Dots: means; light blue area: SEM; blue line: sigmoidal fit $\left(\mathrm{R}^{2}=0.76\right)$.

(D) Free surface of live cells expressing LifeAct-TagRFPT (blue) and E-cadherin-GFP ${ }^{\mathrm{TG}}$ white dotted line: free cell edge; straight white dotted line: site of kymograph; Yellow lines and arrow heads: E-cadherin-containing intracellular vesicles; red lines and arrow heads: E-cadherin surface clusters.

(E) Quantification of E-cadherin motility and actin flow rates in cells as in (D). Intracellular vesicles $(n=94)$, Surface clusters $(n=167)$, Actin flow waves $(n=136)$.

(F) Effect of Blebbistatin, CK666, Jasplakinolide (BCJ) treatment on E-cadherin motility (green) and actin flow rates (magenta). White dotted line in (Fig S1D): site of kymograph.

(G) Quantification of E-cadherin motility and actin flow rates in cells as in (F). Actin flow waves $(n=410)$, E-cadherin clusters $(n=554)$.

(H) Junction formation between cells expressing LifeAct-TagRFPT (magenta) and E-cadherin$\mathrm{GFP}^{\mathrm{TG}}$ (green). Black dotted lines: cell edge of bottom cell; blue arrow heads: E-cadherin surface clusters prior (30 $\mathrm{min})$ and after (34 $\mathrm{min})$ junctional integration; red lines: cluster motility tracks.

$* * * \mathrm{P}<0,001$; Kruskal-Wallis test. Data are means \pm SD with individual datapoints indicated. 
A

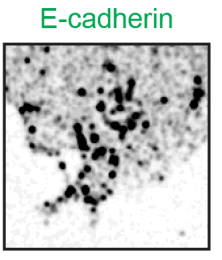

B
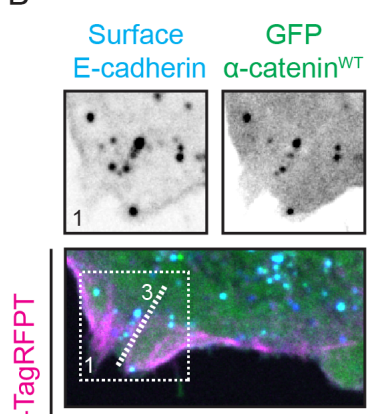

Kymo

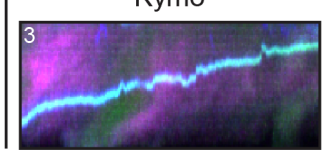

E

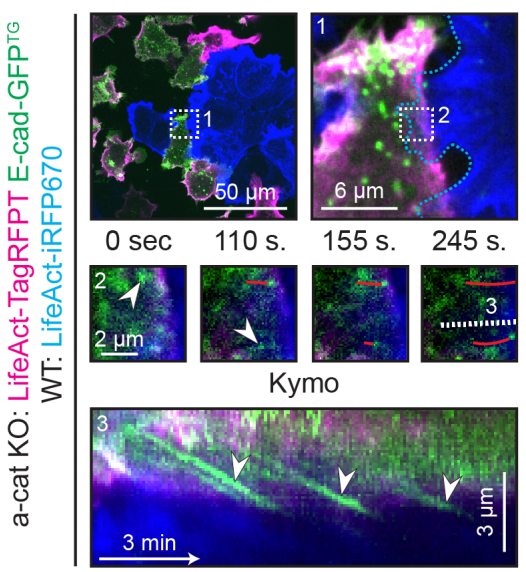

a-catenin

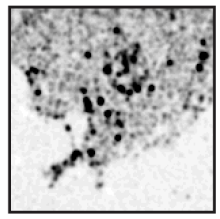

C
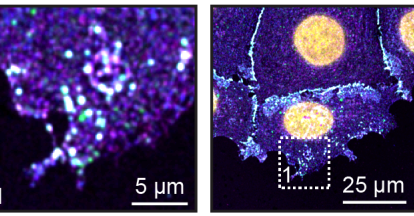

$\mathrm{D}$
Surface GFP

E-cadherin $\alpha$-catenin ${ }^{A+}$
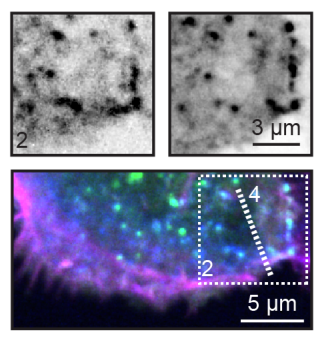

Kymo

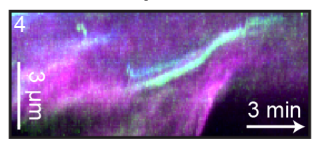

Actin + E-cad/a-cat dynamics
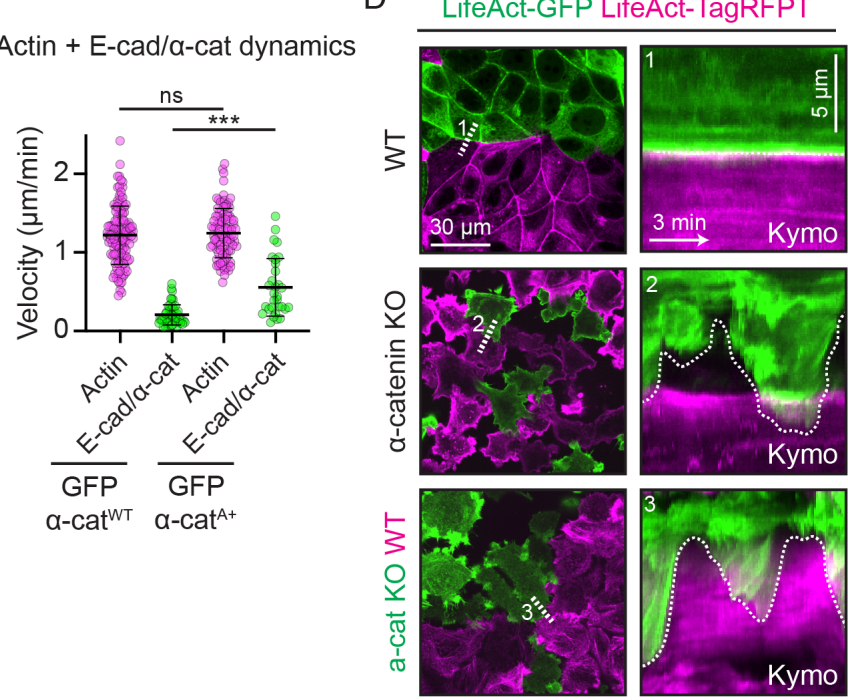

Kymo

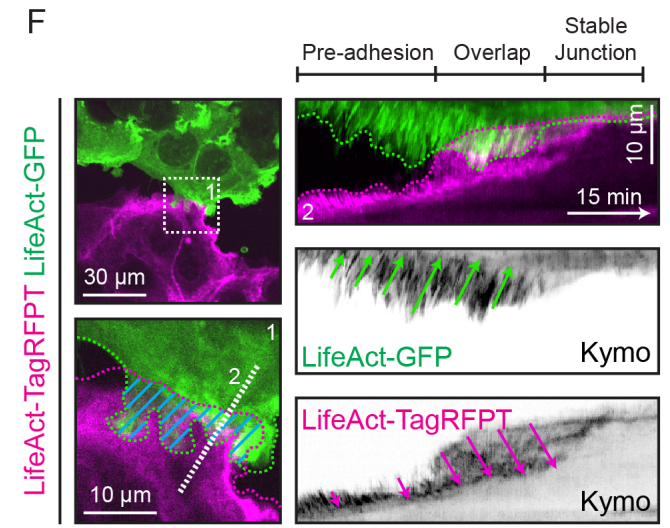

G Actin flow vs.

E-cad accumulation

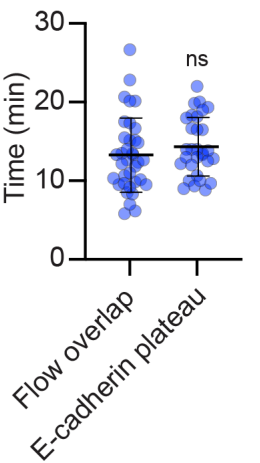

Figure 2. $\alpha$-catenin couples free E-cadherin at the cell surface to the F-actin cortex.

(A) Free edge of MCF7 cells stained for E-cadherin (green), $\beta$-catenin (magenta) and $\alpha$-catenin

(blue). 
(B) Free edge of cells expressing LifeAct-TagRFPT (magenta) and GFP- $\alpha$-catenin ${ }^{\text {WT }}$ or GFP- $\alpha$ catenin $^{\mathrm{A}+}$. Surface E-cadherin is labelled with surface-delivered E-cadherin mAbs (blue). White dotted lines: site of kymographs.

(C) Quantification of actin flows and E-cadherin/ $\alpha$-catenin motility in cells as in (B). Actin ( $\alpha$ cat $\left.^{\mathrm{WT}}\right)$ flow waves $(n=117)$, E-cad$/ \alpha$-cat $\left(\alpha\right.$-cat $\left.{ }^{\mathrm{WT}}\right)$ clusters $(n=49), \operatorname{Actin}\left(\alpha-\right.$ cat $\left.^{\mathrm{A}+}\right)$ flow waves $(n=95), E-c a d / \alpha-$ cat $\left(\alpha-\right.$ cat $\left.^{\mathrm{A}+}\right)$ clusters $(n=31)$.

(D) Confluent layer of Wildtype (WT) cells, $\alpha$-catenin Knockout (KO) cells, or a mixed culture of WT and $\alpha$-catenin KO cells, expressing LifeAct-GFP (green) or LifeAct-TagRFPT (magenta). Wavy white dotted lines: cell edges; straight white dotted lines: site of kymographs. expressing LifeAct-TagRFPT (magenta) and E-cadherin-GFP ${ }^{\mathrm{TG}}$ (green). Blue dotted line: WT cell edge; white dotted line: site of kymograph; white arrow heads: anterograde movement (Kymo) of E-cadherin clusters; red lines: cluster motility tracks.

(F) Junction formation between cells expressing LifeAct-GFP (green) or LifeAct-TagRFPT (magenta). Wavy dotted lines: cell edges; blue lines: overlapping area; green and magenta arrows: direction of actin flows; white dotted line: site of kymograph.

(G) Quantification of overlap phase as in F and the time it takes to reach an E-cadherin plateau as in 1C. Flow overlap during junction formation events $(n=36)$, E-cadherin plateau during junction formation events $(n=30)$.

ns: not significant; $* * * \mathrm{P}<0,001$; Mann-Whitney $\mathrm{U}$ test. Data are means $\pm \mathrm{SD}$ with individual datapoints indicated. 
A

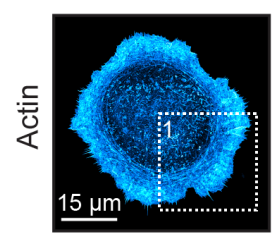

Subnuclear Cortical

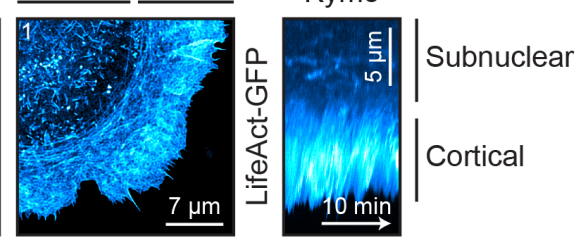

C

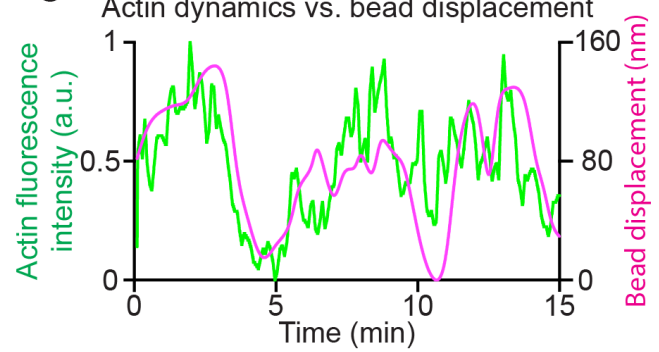

$\mathrm{B}$

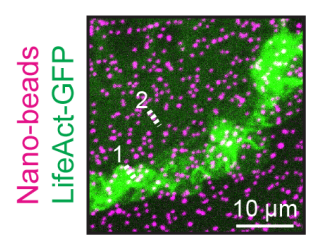

Kymo
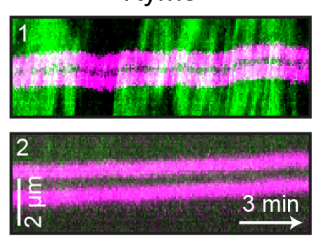

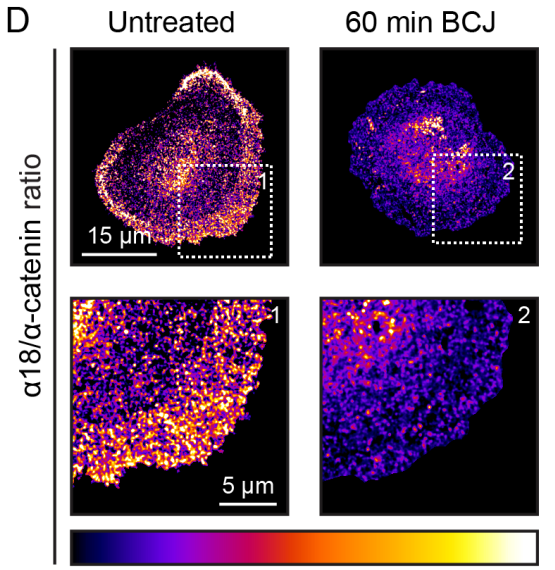

$\rightarrow$ high

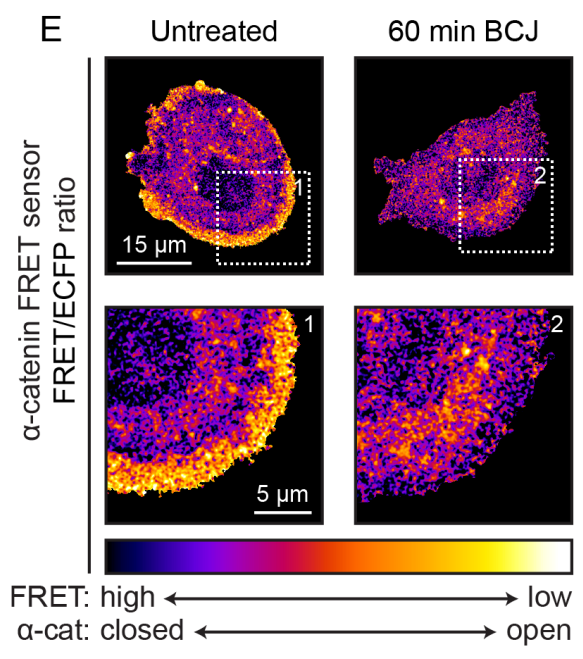

$\mathrm{F}$ a-cat: closed
G

BCJ treatment $+\alpha$-catenin activation

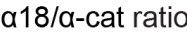

FRET biosensor
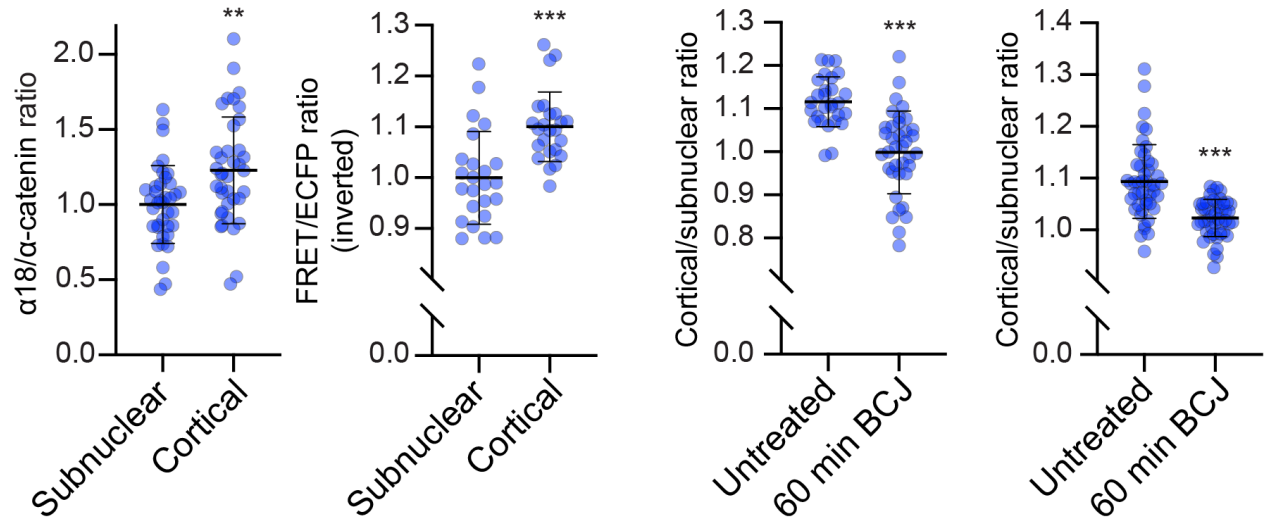

Figure 3. Cortical flow activates cadherin-bound $\alpha$-catenin.

(A) MCF7 cell spread on E-cadherin ectodomain-coated substrate and stained for actin.

Kymograph was generated using a cell expressing LifeAct-GFP (movie S10). 
(B) Cortex of a cell expressing LifeAct-GFP (green), spread on a soft nanobeads-containing (magenta) PDMS substrate (2-3 kPa) coated with E-cadherin ectodomains. White dotted lines: site of kymographs.

(C) Quantification of actin dynamics and bead displacement in kymograph 1 (B).

(D) $\alpha 18 / \alpha$-catenin ratiometric images of cells spread on ectodomain-coated substrata. Images display untreated and BCJ-treated cells.

(E) Ratiometric images of cells expressing $\alpha$-catenin FRET sensor and spread on ectodomaincoated substrata. Images display untreated and BCJ-treated cells.

(F) Quantification of actin flow-mediated $\alpha$-catenin activation in cells spread on E-cadherin ectodomain-coated substrata, using $\alpha 18 / \alpha$-catenin or $\alpha$-catenin FRET sensor ratiometric measurements as in (D+E). $\alpha 18 / \alpha$-cat ratio cells $(n=38)$, FRET biosensor cells $(n=24)$. (G) Quantification of actin flow-mediated $\alpha$-catenin activation in cells spread on E-cadherin ectodomain-coated substrata, using $\alpha 18 / \alpha$-catenin or $\alpha$-catenin FRET sensor ratiometric measurements. Cells are untreated or treated with BCJ as in (D+E). Untreated cells $(\alpha 18 / \alpha-$ catenin ratio) $(\mathrm{n}=28), 60 \mathrm{~min}$ BCJ cells $(\alpha 18 / \alpha$-catenin ratio) $(\mathrm{n}=37)$, Untreated cells (FRET biosensor) ( $\mathrm{n}=46), 60$ min BCJ cells (FRET biosensor) $(\mathrm{n}=46)$.

$* * \mathrm{P}<0,002, * * * \mathrm{P}<0,001$; Mann-Whitney $\mathrm{U}$ test. Data are means $\pm \mathrm{SD}$ with individual datapoints indicated. 
A

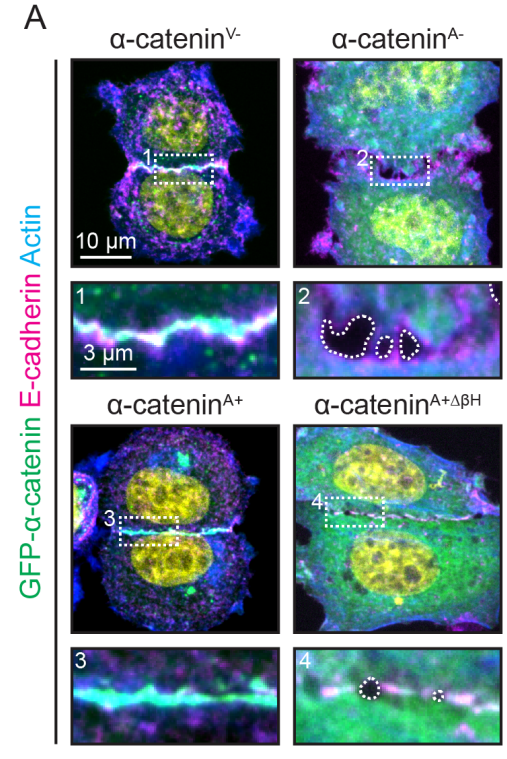

E

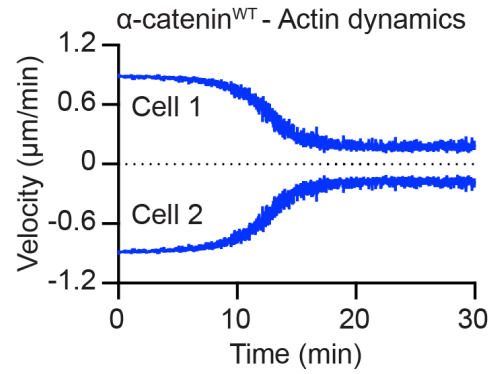

G
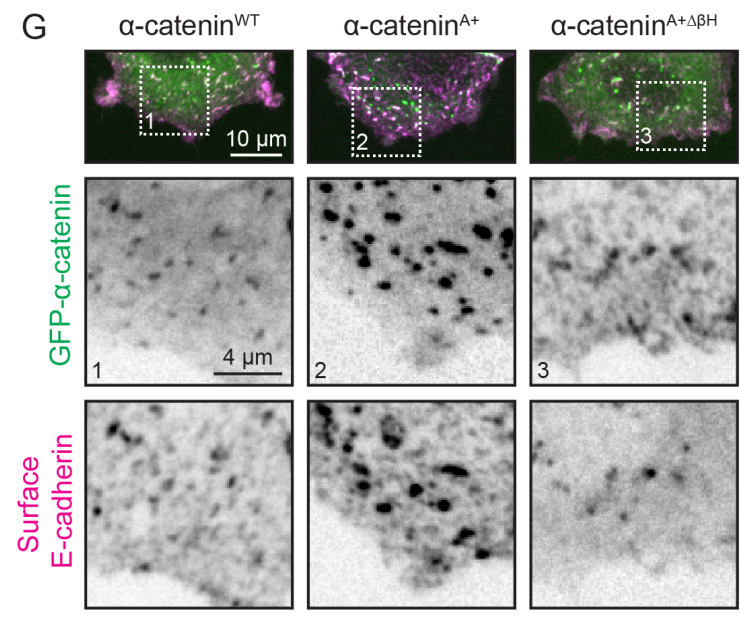

B

C

$\mathrm{F}$

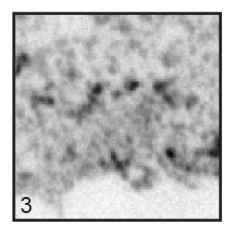

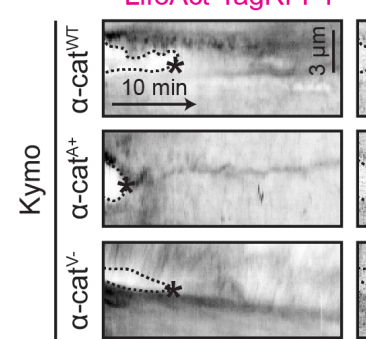
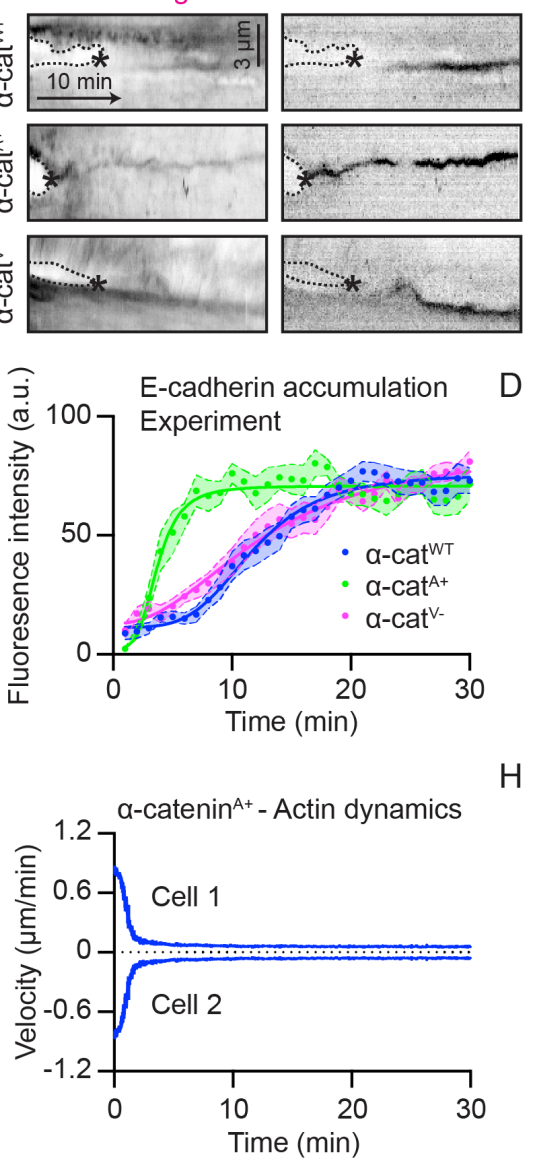

$\mathrm{H}$
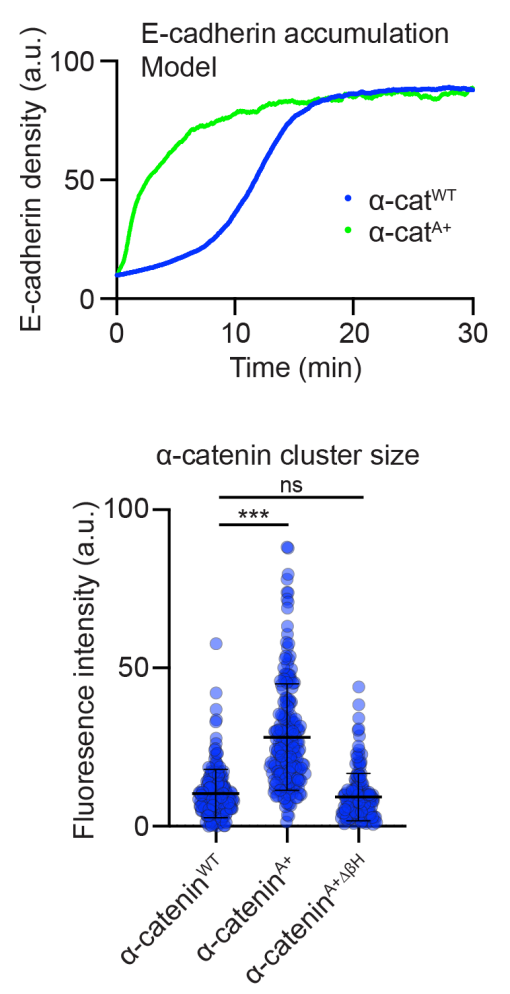

I. Precoupling

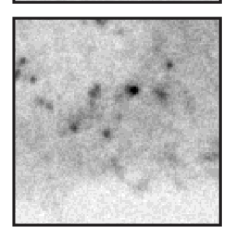

\section{iRFP670-a-cat}
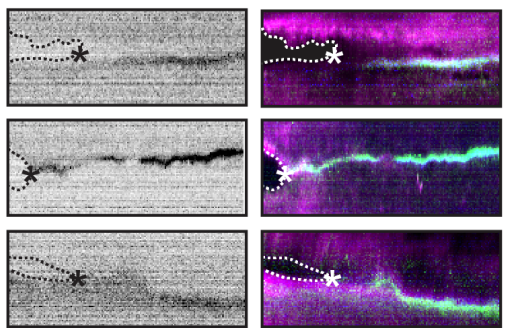

II. Transligation III. Adhesion growth
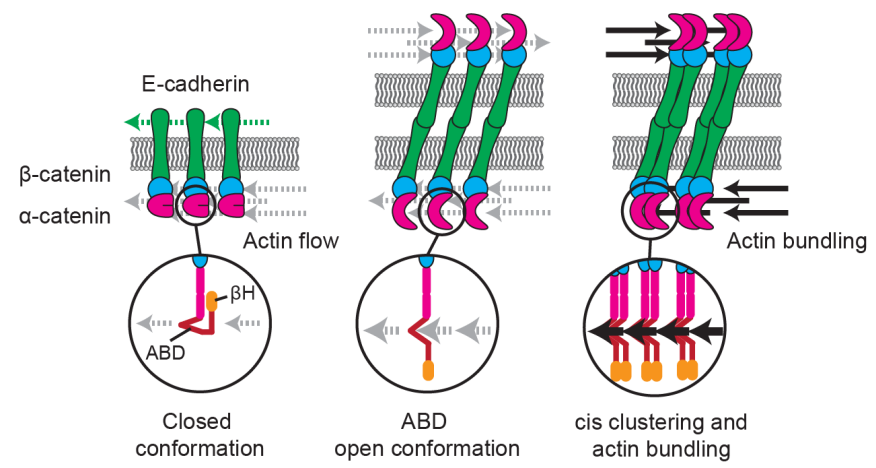

Figure 4. An $\alpha$-catenin clutch stimulates cadherin cis-clustering to assemble adherens

\section{junctions.}


(A) MCF7 cells expressing GFP- $\alpha$-catenin ${ }^{\mathrm{V}-}$, GFP- $\alpha$-catenin ${ }^{\mathrm{A}-}$, GFP- $\alpha$-catenin ${ }^{\mathrm{A}+}$ or GFP- $\alpha-$ catenin $^{\mathrm{A}+\Delta \beta \mathrm{H}}$ (green), stained for E-cadherin (magenta) and actin (blue). White dotted line: free cell edge.

(B) Kymographic analysis of junction formation between cells expressing LifeAct-TagRFPT

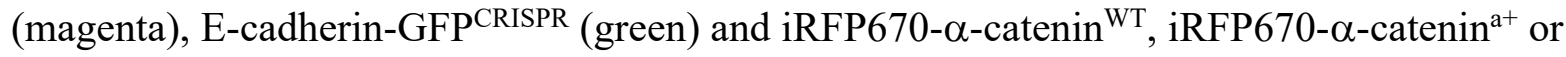
iRFP670- $\alpha$-catenin ${ }^{\mathrm{V}-}$ (blue). Dotted lines: cell edges; *First moment of contact.

(C) Quantification of E-cadherin accumulation during AJ formation in cells as in (B). $\alpha$-cat ${ }^{\mathrm{WT}}$ junction formation events $(n=21), \alpha-$ cat $^{\mathrm{A}+}$ junction formation events $(n=11), \alpha$-cat ${ }^{\mathrm{V}-}$ junction formation events $(n=20)$. Dots: means; light colour areas: SEM; lines: Sigmoidal fits $\left(\alpha-\right.$ cat $^{\text {WT }}$ : $\left.\mathrm{R}^{2}=0,56 ; \alpha-\mathrm{cat}^{\mathrm{A}+}: \mathrm{R}^{2}=0,49 ; \alpha-\mathrm{cat}^{\mathrm{V}-}: \mathrm{R}^{2}=0,52\right)$.

(D) Model predictions for the density of E-cadherin clutches as a function of time. $\alpha$-catenin ${ }^{\mathrm{A}^{+}}$ cells were modelled by increasing the binding rate between cadherin clutches $k_{\text {on }}$, and the amount of cadherin molecules added upon $\alpha 1$ helix unfolding $d_{\text {add }}$ with respect to WT values (Table S1). (E, F) Corresponding model predictions for actin velocities in $\alpha$-catenin WT cells (E) and $\alpha$ 15 catenin ${ }^{\mathrm{A}+}$ cells (F). Top and bottom parts of graphs show antiparallel velocities in each of the two cells making contact.

(G) E-cadherin cis-clusters on free surfaces labelled with extracellular E-cadherin mAbs (magenta) in non-permeabilized cells expressing GFP- $\alpha$-catenin ${ }^{\mathrm{WT}}$, GFP- $\alpha$-catenin ${ }^{\mathrm{A}+}$ or GFP- $\alpha$ catenin $^{\mathrm{A}+\Delta \beta \mathrm{H}}$ (green).

20 (H) Quantification of $\alpha$-catenin cluster intensity in cells as in $(\mathrm{G}) . \alpha$-catenin ${ }^{\mathrm{WT}}$ clusters $(\mathrm{n}=194)$, $\alpha$-catenin ${ }^{\mathrm{A}+}$ clusters $(n=203), \alpha$-catenin ${ }^{\mathrm{A}+\Delta \beta H}$ clusters $(n=158)$.

(I) Schematic overview of how the $\alpha$-catenin clutch induces AJ assembly. I) Surface E-cadherin clusters are coupled to actin retrograde flow through $\beta$ - and $\alpha$-catenin. II) E-cadherin trans- 
ligation results in significant increase in load caused by anti-parallel actin flows. As a result, the $\alpha$-catenin actin binding domain (ABD) changes to an open conformation. III) This conformational change increases the $\alpha$-catenin binding affinity for actin and causes cis-clustering through the $\alpha$-catenin $\beta \mathrm{H}$-domain, resulting in the bundling of actin filaments and growth of the newly formed AJ.

ns: not significant, ${ }^{* * *} \mathrm{P}<0,001$; Kruskal-Wallis test. Data are means $\pm \mathrm{SD}$ with individual datapoints indicated. 